\title{
"Bottom-up" thermalization in heavy ion collisions
}

\author{
R. Baier ${ }^{a}$, A.H. Mueller ${ }^{b}$, D. Schiff ${ }^{c}$, and D.T. Son ${ }^{b, d}$ \\ ${ }^{a}$ Fakultät für Physik, Universität Bielefeld, D-33501 Bielefeld, Germany \\ ${ }^{b}$ Physics Department, Columbia University, New York, NY 10027, USA \\ ${ }^{c}$ LPT, Université Paris-Sud, Bâtiment 210, F-91405 Orsay, France \\ ${ }^{d}$ RIKEN-BNL Research Center, Brookhaven National Laboratory, Upton, NY 11973, USA
}

\begin{abstract}
We describe how thermalization occurs in heavy ion collisions in the framework of perturbative QCD. When the saturation scale $Q_{s}$ is large compared to $\Lambda_{\mathrm{QCD}}$, thermalization takes place during a time of order $\alpha^{-13 / 5} Q_{s}^{-1}$ and the maximal temperature achieved is $\alpha^{2 / 5} Q_{s}$.
\end{abstract}

\section{INTRODUCTION}

It is possible that at RHIC, for the first time, heavy ion collisions occur at energies high enough to be described by perturbative QCD. At the Large Hadron Collider (LHC) perturbative QCD is expected to work even better. At these energies we will assume that immediately after the collision the initial distribution of gluons is given by the saturation scenario [1-6]. Thus the relevant hard scale is the saturation scale $Q_{s}$, estimated to be 1 $\mathrm{GeV}$ at RHIC and 2-3 GeV at LHC.

The single most important question in the physics of heavy ion collisions is thermalization. The conventional argument in favor of thermalization is that at larger collision energy, more gluons are freed in the first moment after the collision, and these gluons collide more frequently with each other. However, the distribution of these gluons is initially very far from thermal equilibrium. In addition, the strong coupling constant decreases at high energies, making thermalization harder to achieve. Whether the system has enough time to equilibrate before falling apart is thus a delicate question requiring detailed consideration of different physical processes.

In this paper we show that, in the limit $Q_{s} \gg \Lambda_{\mathrm{QCD}}$ corresponding to very large nuclei and/or very high collision energy, thermalization occurs relatively fast while the system is still undergoing one-dimensional expansion. The unexpected feature of our analysis is the way thermalization occurs. During the first period of time the most important process is the emission of soft gluons which overwhelm, in terms of number, the primary hard gluons at time $\tau \sim \alpha^{-5 / 2} Q_{s}^{-1}$. These soft gluons then quickly equilibrate and form a thermal bath, which initially carries only a small fraction of the total energy. The thermal bath then draws energy from the hard gluons. Full thermalization is achieved when the primary hard gluons have lost all their energy. Parametrically, this happens at $\tau \sim \alpha^{-13 / 5} Q_{s}^{-1}$, at which time the temperature of the system achieves the maximal value of $\alpha^{2 / 5} Q_{s}$. Surprisingly, the 
time dependence of the temperature of the soft sector in the (admittedly narrow) region $\alpha^{-5 / 2} \ll Q_{s} \tau \ll \alpha^{-13 / 5}$ can be found almost analytically, and the result depends only on the total number of primary hard gluons. It is important to emphasize here that we only provide parametric qualitative and quantitative estimates based on the assumption that always $\alpha \ll 1$. A more realistic study is required in order to figure out the numerical coefficients of the given estimates.

This picture of "bottom up" thermalization is different from that considered in a previous work by one of us [7] (see also [8]) which did not take into account particle production, and also of Refs. [9 14]. In [1] the importance of inelastic processes, even for kinetic equilibration, has been observed when using the relaxation time approximation for the collision term and with initial conditions different from the ones considered here. Although the analysis requires a small coupling, one can hope that many qualitative features of this picture are already present in heavy ion collisions at LHC or even RHIC energies. At the very least, the finding gives us confidence that thermalization always occurs in heavy ion collisions at sufficiently high energies. Our main emphasis here is the thermalization that begins with the softer momentum modes. Still more detailed investigations, including more specific realistic initial conditions, are required for treating the high momentum tails of the distributions which are expected to require a longer thermalization time, but which are important for plasma signatures sensitive to momentum scales larger than $Q_{s}$.

\section{QUALITATIVE DESCRIPTION OF EARLY TIMES}

We will be interested only in the central rapidity region of central collisions. In this region one can assume boost invariance: all physical quantities depend only on the proper time $\tau=\sqrt{t^{2}-z^{2}}$, but not on the rapidity $\eta=\frac{1}{2} \ln \frac{t+z}{t-z}$, where $z$ is the direction of collision. For large nuclei, the dependence on the transverse ( $x$ and $y$ ) coordinates can also be neglected, which is equivalent to assuming the medium to be infinite in the transverse directions. With these simplifications, all physical quantities depend only on the single coordinate $\tau$. The evolution proceeds through several regimes, with $Q_{s} \tau \sim \alpha^{-3 / 2}, \alpha^{-5 / 2}$, and $\alpha^{-13 / 5}$ marking the borders between neighboring time periods.

We will describe qualitatively the evolution of the system up to $Q_{s} \tau \sim \alpha^{-5 / 2}$. In all subsequent estimates, we will assume $\ln \frac{1}{\alpha} \sim 1$ and keep track only of powers of $\alpha$ itself.

\section{A. Very early time, $1 \ll Q_{s} \tau \ll \alpha^{-3 / 2}$}

At the earliest time, $\tau \sim Q_{s}^{-1}$, gluons are freed from the nuclei. These gluons have typical momentum of order $Q_{s}$ and occupation number of order $1 / \alpha$. This is the basic assumption of the rest of this paper. Due to the large occupation number these gluons interact so strongly that it is more appropriate to describe them as a nonlinear gluon field rather than a collection of particles. Only when $Q_{s} \tau$ becomes larger than 1, the classical field becomes almost linear, and one can start to describe the gluons as particles on mass shell with a well-defined distribution, 


$$
\frac{d N}{d y d k_{\perp}^{2}}=\frac{1}{\alpha} f\left(\frac{k_{\perp}}{Q_{s}}\right)
$$

The precise form of $f$ depends on the details of the nonperturbative physics at the scale $\tau \sim Q_{s}^{-1}$ and is not a subject of the present paper. A promising method to find $f$ is by simulation of the classical gluon field [15]. We will call the particles produced during these first periods the hard gluons, since later gluons with smaller momenta will be produced.

The density of hard gluons [1]6] decreases with time due to the one-dimensional expansion,

$$
N_{h} \sim \frac{Q_{s}^{3}}{\alpha\left(Q_{s} \tau\right)}
$$

If there was no interaction, the occupation number would remain of order $1 / \alpha$ due to the Liouville theorem. There is no contradiction with Eq. (2), since the typical longitudinal momentum of hard gluons also becomes smaller $\left(p_{z} \sim 1 / \tau\right)$ because gluons with larger longitudinal momentum escape from the spatial region under consideration during a time of order $\tau$.

In reality, gluons interact by elastic scattering. Most of the scatterings are small angle, with exchange momentum $q \ll Q_{s}$. The effect is the broadening of the distribution along the $p_{z}$ direction, thus lowering the typical occupation number. The lowest possible momentum exchange is the Debye mass, which is determined by [16,8, 17]

$$
m_{\mathrm{D}}^{2} \sim \alpha \int d^{3} p \frac{f_{h}(p)}{p} \sim \frac{\alpha N_{h}}{Q_{s}} \sim \frac{Q_{s}^{2}}{Q_{s} \tau} .
$$

If one assumes that $m_{\mathrm{D}} \ll p_{z}$ (this condition will be verified a posteriori), most collisions do not take particles away from the momentum region where the occupation number is large $\left(k_{\perp} \sim Q_{s}, k_{z} \sim p_{z}\right)$. The frequency of collisions that a typical particle encounters is enhanced by the Bose factor,

$$
\frac{d N_{\mathrm{col}}}{d \tau} \sim \sigma N_{h}\left(1+f_{h}\right) \sim \frac{\alpha N_{h}}{m_{\mathrm{D}}^{2} p_{z} \tau},
$$

where $\sigma \sim \alpha^{2} m_{\mathrm{D}}^{-2}$ is the cross section, and $f_{h}=N_{h} /\left(Q_{s}^{2} p_{z}\right)$ is the typical occupation number, which is assumed to be large. These random collisions increase the longitudinal momentum of gluons, which is typically

$$
p_{z}^{2} \sim N_{\mathrm{col}} m_{\mathrm{D}}^{2} \sim \frac{\alpha N_{h}}{p_{z}}
$$

which implies

$$
p_{z} \sim\left(\alpha N_{h}\right)^{1 / 3} \sim \frac{Q_{s}}{\left(Q_{s} \tau\right)^{1 / 3}}
$$

This relation may be derived from [7], taking the Bose enhancement into account, which amounts to replace the average $p_{z}^{2} \sim \alpha Q_{s}^{2}$ by $p_{z}^{2} \sim \alpha_{s} Q_{s}^{2}\left(1+f_{h}\right) \sim \alpha N_{h} / p_{z}$. ¿From Eqs. 
(3) and (6) we see that $m_{\mathrm{D}} \ll p_{z}$, as assumed. The typical occupation number $f_{h} \sim$ $\alpha^{-1}\left(Q_{s} \tau\right)^{-2 / 3}$ is large until $Q_{s} \tau \sim \alpha^{-3 / 2}$.

Beside elastic scatterings there are also inelastic scatterings in which gluons are produced. As we will see, the most important produced gluons are those with smallest energies. In principle gluons with energy as low as $m_{\mathrm{D}}$ can be produced. However, once produced, the momentum of these gluons is pushed up by multiple elastic scatterings with hard gluons. As a consequence of (6) the smallest momentum of soft gluons is of order $p_{z}: k_{s} \sim p_{z} \sim$ $Q_{s} /\left(Q_{s} \tau\right)^{1 / 3}$. The number of soft gluons with momentum $k_{s}$ at time $\tau$ that are produced at this moment is estimated from the Bethe-Heitler formula to be [18]

$$
N_{s} \sim \tau \frac{\partial N_{s}}{\partial \tau} \sim \tau \int d^{3} p f(p) \frac{d I^{\mathrm{BH}}}{d t}\left(1+f_{h}\right)^{2} \sim \tau \frac{\alpha^{3}}{m_{\mathrm{D}}^{2}} N_{h}^{2}\left(1+f_{h}\right)^{2} \sim \frac{Q_{s}^{3}}{\alpha\left(Q_{s} \tau\right)^{4 / 3}}
$$

The time interval around the moment $\tau$ is taken to be of order $\tau$. Once these soft gluons are produced they will remain, and their density is decreasing as $1 / \tau$. One notices that $N_{s} / k_{s} \sim N_{h} / Q_{s}$, so the Debye mass receives equal contributions from hard and soft gluons,

$$
m_{\mathrm{D}}^{2} \sim \frac{\alpha N_{h}}{Q_{s}}+\frac{\alpha N_{s}}{k_{s}}
$$

but the estimate of Eq. (3) is still valid parametrically.

\section{B. Setting up the stage for thermalization: $\alpha^{-3 / 2} \ll Q_{s} \tau \ll \alpha^{-5 / 2}$}

Beginning from $Q_{s} \tau \sim \alpha^{-3 / 2}$, the occupation number of hard gluons drops below 1 , and the estimates of the previous section need to be revised. We will see that when $\alpha^{-3 / 2} \ll$ $Q_{s} \tau \ll \alpha^{-5 / 2}$ soft gluons contribute negligibly to the total number of gluons but give most of the Debye screening. In other words,

$$
\begin{gathered}
N_{s} \ll N_{h}, \\
m_{\mathrm{D}}^{2} \sim \frac{\alpha N_{s}}{k_{s}} .
\end{gathered}
$$

First let us estimate $k_{s}$, which is the typical momentum of soft gluons. When $f_{h} \ll 1$, we have

$$
k_{s}^{2} \sim N_{\mathrm{col}} m_{\mathrm{D}}^{2} \sim \tau \sigma N_{h} m_{\mathrm{D}}^{2} \sim \alpha Q_{s}^{2},
$$

which is now a constant scale. The number of soft gluons that have been produced at time $\tau$ is

$$
N_{s} \sim \tau \frac{\alpha^{3}}{m_{\mathrm{D}}^{2}} N_{h}^{2} \sim \frac{\alpha Q_{s}^{4}}{m_{\mathrm{D}}^{2} \tau} .
$$

¿From Eqs. (9) and (11), one finds 


$$
\begin{gathered}
N_{s} \sim \frac{\alpha^{1 / 4} Q_{s}^{3}}{\left(Q_{s} \tau\right)^{1 / 2}}, \\
m_{\mathrm{D}} \sim \frac{\alpha^{3 / 8} Q_{s}}{\left(Q_{s} \tau\right)^{1 / 4}} .
\end{gathered}
$$

For the soft gluons to give the dominant contribution to Debye screening one needs $N_{s} / k_{s} \gg$ $N_{h} / Q_{s}$, which requires $Q_{s} \tau \gg \alpha^{-3 / 2}$. The number of soft gluons become comparable to that of hard ones at $Q_{s} \tau \sim \alpha^{-5 / 2}$. Therefore all estimates in this section are valid in the interval $\alpha^{-3 / 2} \ll Q_{s} \tau \ll \alpha^{-5 / 2}$.

In-medium emission of gluons is suppressed by the Landau-Pomeranchuk-Migdal (LPM) effect. The latter is operative at scales larger than $k_{\mathrm{LPM}}$ determined by $k_{\mathrm{LPM}}=m_{\mathrm{D}}^{2}\left(N_{h} \sigma\right)^{-1}$ [19,20, or

$$
k_{\mathrm{LPM}} \sim \frac{m_{\mathrm{D}}^{4}}{\alpha^{2} N_{h}} \sim \alpha^{1 / 2} Q_{s}
$$

which is parametrically of the same order as the scale $k_{s}$. The LPM effect suppresses the gluon production compared to the Bethe-Heitler rate at scales larger than $k_{s}$, but does not affect the rate of producing particles in the interval between $m_{\mathrm{D}}$ and $k_{s}$. Therefore all previous estimates based on the Bethe-Heitler formula remain intact.

\section{THERMALIZATION OF THE SOFT SECTOR: $Q_{s} \tau \gg \alpha^{-5 / 2}$}

\section{A. Qualitative description}

After $Q_{s} \tau \sim \alpha^{-5 / 2}$ most gluons are soft, $N_{s} \gg N_{h}$. We will see that the soft gluons collide very frequently with each other, and achieve thermal equilibration amongst themselves. The soft sector is characterized by the temperature $T$, which is a function of time. The system as a whole is still not in thermal equilibrium, since most of the energy is carried by a small number of hard gluons. These few gluons collide with the soft gluons of thermal bath and constantly loose energy to the latter.

A hard gluon with energy of order $Q_{s}$ looses energy to the bath by the following mechanism. First it emits a particle with a softer momentum $k_{\mathrm{br}}$, which, during a time comparable to $\tau$, splits into two gluons with comparable momenta (hard branching). The products of this branching quickly cascade further, giving all their energy to the thermal bath.

As we will verify, $k_{\mathrm{br}}$ lies in the region where the emission rate is LPM-suppressed. One can estimate the time of emission, $t_{\mathrm{br}}$, for a gluon having momentum $k_{\mathrm{br}}$ as follows: $1 / t_{\mathrm{br}} \sim$ $\alpha / t_{\mathrm{f}}$, with the formation time $t_{\mathrm{f}}$ given by $t_{\mathrm{f}} \sim k_{\mathrm{br}} / k_{t}^{2}$. The gluon picks up a momentum $k_{t}$, transverse to its direction of motion, given by $k_{t}^{2} \sim m_{\mathrm{D}}^{2} t_{\mathrm{f}} / \lambda$. Using $\lambda^{-1} \sim N_{s} \sigma \sim N_{s} \alpha^{2} / m_{\mathrm{D}}^{2}$ one obtains (see also Eq. (19) below),

$$
\frac{1}{t_{\mathrm{br}}} \sim \frac{\alpha^{2} N_{s}^{1 / 2}}{k_{\mathrm{br}}^{1 / 2}} .
$$


Equating the branching time $t_{\mathrm{br}}$ with $\tau$, and using $N_{s} \sim T^{3}$, where $T$ is the temperature of the soft thermal bath, we find that $k_{\mathrm{br}} \sim \alpha^{4} T^{3} \tau^{2}$. The time dependence of the temperature $T$ is still to be found.

The number of $k_{\mathrm{br}}$-gluons produced per unit time per unit volume is

$$
\frac{d N\left(k_{\mathrm{br}}\right)}{d \tau} \sim \frac{N_{h}}{t_{\mathrm{br}}} \sim \frac{\alpha^{2} N_{s}^{1 / 2} N_{h}}{k_{\mathrm{br}}^{1 / 2}} \sim \frac{Q_{s}^{2}}{\alpha \tau^{2}} .
$$

Subsequently, the rate of energy flow from the hard gluons to the soft thermal bath is

$$
k_{\mathrm{br}} \frac{d N\left(k_{\mathrm{br}}\right)}{d \tau} \sim \alpha^{3} Q_{s}^{2} T^{3}
$$

This energy flow increases the energy in the thermal bath, and thus must be proportional to $d\left(T^{4}\right) / d \tau$. Therefore one finds

$$
T \sim \alpha^{3} Q_{s}^{2} \tau
$$

The temperature of the soft thermal bath increases linearly with time, even when the system is expanding, due to the hard gluons which serve as an energy source. When the bath starts forming $\left(Q_{s} \tau \sim \alpha^{-5 / 2}\right)$, its temperature is $\alpha^{1 / 2} Q_{s}$. The relaxation time of the soft sector is of order $\tau_{\text {rel }} \sim\left(\alpha^{2} T\right)^{-1} \sim\left(\alpha^{5} Q_{s}^{2} \tau\right)^{-1}$. When $Q_{s} \tau \gg \alpha^{-5 / 2}, \tau_{\text {rel }} \ll \tau$, which justifies the assumption of thermal equilibration of the soft sector, i.e. in the relaxation time approximation $f_{s} \rightarrow f^{\mathrm{eq}}\left(1-\exp \left(-\tau / \tau_{\text {rel }}\right)\right)$.

The linear growth of $T$ terminates when the hard gluons loose all of their energy. This happens when $k_{\mathrm{br}} \sim Q_{s}$, or $\tau \sim \alpha^{-13 / 5} Q_{s}^{-1}$, when the temperature achieves a maximal value of order $\alpha^{2 / 5} Q_{s}$, which is larger than the initial temperature only by a factor of $\alpha^{-1 / 10}$. Subsequently the temperature decreases as $\tau^{-1 / 3}$ [22].

The border between the Bethe-Heitler and LPM regimes is at $k_{\mathrm{LPM}}=m_{\mathrm{D}}^{4} /\left(\alpha^{2} N_{s}\right) \sim$ $T \sim \alpha^{3} Q_{s}^{2} \tau$. On the other hand $k_{\mathrm{br}} \sim \alpha^{4} T^{3} \tau^{2} \sim \alpha^{13} Q_{s}^{6} \tau^{5}$, which is much larger than $k_{\mathrm{LPM}}$ when $Q_{s} \tau \gg \alpha^{-5 / 2}$. Thus the use of the LPM formula in Eq. (13) is justified.

\section{B. Quantitative description}

The picture given above can made quantitative in the form of a Boltzmann equation describing the kinetics of the branching process. In this kinetic approach the evolution of hard modes follows the equation:

$$
\left(\frac{\partial}{\partial \tau}-\frac{p_{z}}{\tau} \frac{\partial}{\partial p_{z}}\right) f(\vec{p})=C_{\mathrm{el}}+C_{\text {prod }}
$$

where $C_{\mathrm{el}}$ is the elastic collision integral (see Ref. [7]) and

$$
\begin{aligned}
C_{\text {prod }}= & \int_{0}^{1} d x \frac{d^{2} I}{d x d t}\left\{\frac{1}{x^{5 / 2}}\left[f\left(\frac{\vec{p}}{x}\right)(1+f(\vec{p}))\left(1+f\left(\frac{\vec{p}(1-x)}{x}\right)\right)-f(\vec{p}) f\left(\frac{\vec{p}(1-x)}{x}\right)\left(1+f\left(\frac{\vec{p}}{x}\right)\right)\right]\right. \\
& \left.-\frac{1}{2}[f(\vec{p})(1+f(\vec{p} x))(1+f(\vec{p}(1-x)))-f(\vec{p} x) f(\vec{p}(1-x))(1+f(\vec{p}))]\right\}
\end{aligned}
$$


is the term describing the $2 \rightarrow 3$ and $3 \rightarrow 2$ processes. The four terms in the collision integral correspond to the diagrams (a), (c), (b) and (d) in Fig. 1. The black blob in this Figure represents multiple scatterings off individual gluons in the medium. In Eq. (18) $d^{2} I / d x d t$ is the rate of a hard gluon with momentum $\vec{p} \sim Q_{s}$ to split almost collinearly into two gluons with momenta $\vec{p} x$ and $\vec{p}(1-x)$ while moving in a medium where most particles carry much smaller momenta. This rate is LPM-suppressed and can be computed using the method of Ref. [19 21], for an infinite size medium, which yields

$$
\frac{d^{2} I}{d x d t}=\frac{\alpha^{2} N^{1 / 2}}{p^{1 / 2}} h(x)
$$

where

$$
h(x)=h_{0} \frac{\left(1-x+x^{2}\right)^{5 / 2}}{\left(x-x^{2}\right)^{3 / 2}}, \quad h_{0}=\frac{2}{\pi^{1 / 2}} \frac{N_{c}^{2}}{\left(N_{c}^{2}-1\right)^{1 / 2}}\left(\ln \frac{\left\langle k_{t}^{2}\right\rangle}{m_{\mathrm{D}}^{2}}\right)^{1 / 2}
$$

and

$$
N=2\left(N_{c}^{2}-1\right) \int \frac{d \vec{p}}{(2 \pi)^{3}} f(\vec{p})(1+f(\vec{p}))
$$

The $N$ in (21) represents the density of possible scatterers in the system. $N$ is not quite the same as the density of gluons because of the extra factor of $(1+f)$, a factor necessary to correctly give the interaction rate. From the discussion given earlier we expect $N_{s} \gg N_{h}$ so long as $Q_{s} \tau>\alpha^{-5 / 2}$ and so we expect $N$ to be dominated by the soft (thermalized) particles in our present discussion.

$h(x)$ has a symmetry property that $h(x)=h(1-x)$, using which one can check that the collision term (18) conserves energy, $\int d \vec{p} p C_{\text {prod }}(p)=0$. One might wonder why $C_{\text {prod }}$ is of order $\alpha^{2}$ instead of $\alpha^{3}$. An intuitive derivation for this dependence is already given just above Eq. (13).

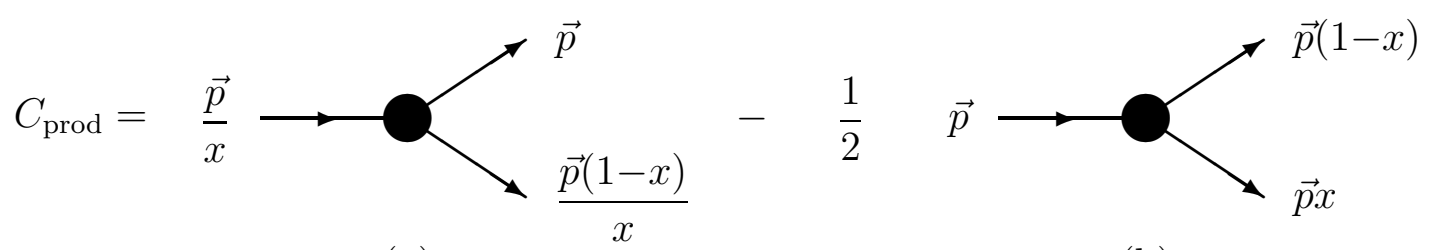

(a)

(b)

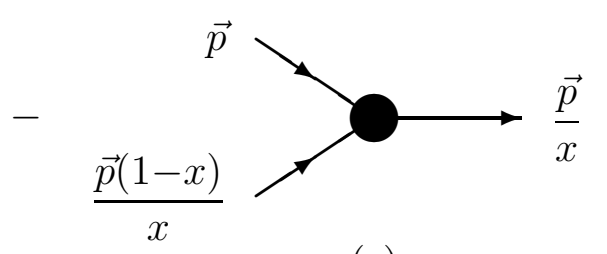

(c)

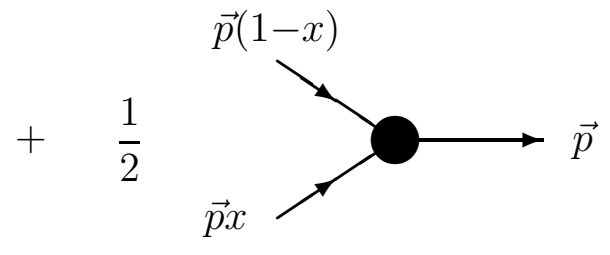

(d)

FIG. 1. The diagrammatic representation of $C_{\text {prod }}$ 
A remark on the Boltzmann equation is in order. In Ref. [7] a Boltzmann equation without particle production was considered. The system does seem to approach kinetic equilibration [0, 8], but during a relatively long time $\left(\sim \exp \left(\alpha^{-1 / 2}\right) Q_{s}^{-1}\right)$. This is because most of the elastic scatterings are at small angle, and it takes a lot of small angle scatterings to change the particle distribution considerably. In more technical terms, there is a cancellation between gain and loss terms in the elastic collision integral. The $2 \rightarrow 3$ process, on the other hand, is asymmetric and does not have this type of cancellation, and so is important although it is smaller than the elastic rate. Parametrically higher-order processes like $2 \rightarrow 4$ can be neglected since they are suppressed by an additional factor of $\alpha$ compared to $2 \rightarrow 3$ while not adding any qualitatively new feature to the evolution. Whether in a realistic circumstance, say at RHIC, higher order processes are negligible or not is beyond the scope of the present discussion [10].

The elastic collision integral $C_{\mathrm{el}}$ is responsible for the thermalization of the soft sector $p \sim T$, and for $p_{z}$ broadening of the hard particles. The inelastic term $C_{\text {prod }}$ is responsible for the energy flow from the hard to the soft sector. In principle, the products of branching of hard gluons have a small transverse momentum, but this is negligible compared to the subsequent broadening by elastic scattering. Therefore, $C_{\text {prod }}$ is written as if the branching was exactly collinear.

For hard gluons which have small occupation number $(f \ll 1)$ Eq. (17) simplifies considerably. If we introduce

$$
\epsilon\left(p_{\perp}\right)=2 \pi p_{\perp}^{2} \int d p_{z} f(\vec{p})
$$

which is normalized so that the energy carried by hard gluons is $\int d p_{\perp} \epsilon\left(p_{\perp}\right)$, then the kinetic equation for $\epsilon\left(p_{\perp}\right)$ becomes

$$
\frac{1}{\tau} \frac{\partial}{\partial \tau}\left(\tau \epsilon\left(p_{\perp}\right)\right)=\frac{\alpha^{2} N^{1 / 2}}{p_{\perp}^{1 / 2}} \int d x h(x)\left[x^{1 / 2} \epsilon\left(\frac{p_{\perp}}{x}\right)-\frac{1}{2} \epsilon\left(p_{\perp}\right)\right] .
$$

In the following we will write $p$ instead of $p_{\perp}$ for simplicity. Eq. (23) does not describe the soft gluons, for which $C_{\mathrm{el}}$ is essential. Fortunately, these gluons are fully equilibrated and one can characterize the soft sector by the temperature $T$. In particular,

$$
N=g_{N} T^{3}, \quad g_{N}=2\left(N_{c}^{2}-1\right) \frac{1}{6} .
$$

The time evolution of $T$ depends on the amount of energy that flows from the hard to the soft sector. To find this quantity, let us introduce an intermediate scale $p_{0}, T \ll p_{0} \ll Q_{s}$, and integrate Eq. (23) from $p_{0}$ to $\infty$. One finds

$$
\frac{1}{\tau} \frac{\partial}{\partial \tau}\left(\tau \int_{p_{0}}^{\infty} d p \epsilon(p)\right)=-\alpha^{2} N^{1 / 2} \int_{0}^{1} d x h(x) x \int_{p_{0}}^{p_{0} / x} d p \frac{\epsilon(p)}{p^{1 / 2}} .
$$

The left hand side of Eq. (25) has the meaning of the rate of energy flow from above $p_{0}$ to below $p_{0}$. This quantity must be a constant independent of $p_{0}$ when $p_{0} \ll Q_{s}$. This is possible only when $\epsilon(p)$ has the following behavior at small $p$ : 


$$
\epsilon(p)=\frac{\epsilon_{1}}{p^{1 / 2}}, \quad T \ll p \ll Q_{s} .
$$

The right hand side of Eq. (25) is then $-b h_{0} \alpha^{2} N^{1 / 2} \epsilon_{1}$, where

$$
b=\int_{0}^{1} d x \frac{\left(1-x+x^{2}\right)^{5 / 2}}{\left(x-x^{2}\right)^{3 / 2}} x \ln \frac{1}{x} \approx 4.96 .
$$

The equation that governs the evolution of the temperature is then

$$
\frac{1}{\tau^{4 / 3}} \frac{\partial}{\partial \tau}\left(\tau^{4 / 3} \epsilon_{s}(T)\right)=b h_{0} \alpha^{2} N^{1 / 2} \epsilon_{1}
$$

because of the one-dimensional expansion [22,23] where $\epsilon_{s}(T)$ is the energy density of the soft gluons at temperature $T$,

$$
\epsilon_{s}(T)=g_{E} T^{4}, \quad g_{E}=2\left(N_{c}^{2}-1\right) \frac{\pi^{2}}{30}
$$

Eqs. (23), (24), (28), and (29) are the equations that govern the evolution after $Q_{s} \tau \sim \alpha^{-5 / 2}$. From Eq. (23) one needs to extract $\epsilon_{1}$, which enters Eq. (28) that describes the temperature $T$, which feeds back to Eq. (23) through $N$.

Let us show that this procedure can be performed analytically in the regime $\alpha^{-5 / 2} \ll$ $Q_{s} \tau \ll \alpha^{-13 / 5}$. Eq. (23) can be solved by iteration. We will be looking for the solution in the form

$$
\epsilon(\tau, p)=\epsilon_{0}(\tau, p)+\epsilon_{1}(\tau, p)+\epsilon_{2}(\tau, p)+\cdots
$$

where $\epsilon_{0}(\tau, p)$ is the energy distribution which starts the iteration. It is related to the number of hard gluons by

$$
N_{h}(\tau)=\int_{p}^{\infty} d k k^{-1} \epsilon_{0}(\tau, k)
$$

so long as $p \gg T$ and $p \ll Q_{s}$. Due to the one-dimensional expansion it decreases as $\mathbf{1} / \boldsymbol{\tau}$. Solving for $\epsilon_{1}(\tau, p)$, we find, for $p \ll Q_{s}$,

$$
\epsilon_{1}(\tau, p)=\frac{\epsilon_{1}(\tau)}{p^{1 / 2}}
$$

where $\epsilon_{1}(\tau)$ satisfies the equation

$$
\frac{\partial}{\partial \tau}\left(\tau \epsilon_{1}(\tau)\right)=\alpha^{2} h_{0} N^{1 / 2} N_{h} \tau
$$

We see that $\epsilon_{1}(\tau, p)$ is singular at small $p$, and hence at very small $p, \epsilon_{1} \gg \epsilon_{0}$. One could expect that at these small $p$ 's the series $(30)$ is ill behaved. However, if one tries to do the second iteration and inserts (32) into the collision integral of Eq. (23), the result is zero. This is because $\epsilon(p)=\mathrm{const} / p^{1 / 2}$ is a formal static solution to the Boltzmann equation 
(23). This solution describes a stationary state with a constant energy flow from high to low momenta. As a consequence, at small $p$ the iteration procedure stops at the first iteration, and the solution to the Boltzmann equation is simply $\epsilon_{0}+\epsilon_{1}$. Notice that Eq. (32) has the form expected in Eq. (26).

To solve Eqs. (33) and (28) one needs to know only the total number of the hard gluons, $N_{h}$, but not the whole distribution function. This number can be parametrized by $Q_{s}$ and a dimensionless constant $c \| 7]$ :

$$
N_{h}=\frac{N_{c}^{2}-1}{4 \pi^{2} N_{c}} c \frac{Q_{s}^{3}}{\alpha\left(Q_{s} \tau\right)} .
$$

Eqs. (33) and (28) then can be solved. For the temperature, we have $T=c_{T} \alpha^{3} Q_{s}^{2} \tau$, where

$$
c_{T}=\frac{3}{160 \pi^{2}} \frac{N_{c}^{2}-1}{N_{c}} \frac{g_{N}}{g_{E}} b h_{0}^{2} c=\frac{3 b}{8 \pi^{5}} N_{c}^{3} c \ln \frac{\left\langle k_{t}^{2}\right\rangle}{m_{\mathrm{D}}^{2}} .
$$

Eq. (35) is valid only with logarithmic accuracy. This is due to the fact that the rate of branching by a hard particle in a thermal medium $d^{2} I / d x d t$ is known only with this accuracy. Since $k_{t}^{4} \sim \alpha^{2} N_{s} k_{\mathrm{br}}, k_{t}^{2} / m_{\mathrm{D}}^{2} \sim \alpha^{5}\left(Q_{s} \tau\right)^{2}$, and the argument of the logarithm in Eq. (35) is parametrically large for $Q_{s} \tau \gg \alpha^{-5 / 2}$. An improved calculation of $d^{2} I / d x d t$ would give a more accurate estimate for $T$ by precisely determining the constant in the logarithm in Eq. (35). Thus we arrive at the same results, with a more accurate determination of $T$, as in part A of this section.

The authors are indebted to M. Gyulassy, L. McLerran, and R. Venugopalan for discussions. DTS thanks RIKEN, Brookhaven National Laboratory, and U.S. Department of Energy [DE-AC02-98CH10886] for providing the facilities essential for the completion of this work. RB acknowledges support, in part, by DFG, contract Ka 1198/4-1. The work of AHM is supported, in part, by a DOE Grant. The work of DTS is supported, in part, by a DOE OJI Award. 


\section{REFERENCES}

[1] L.V. Gribov, E.M. Levin, M.G. Ryskin, Phys. Rep. 100 (1983) 1.

[2] J.-P. Blaizot, A.H. Mueller, Nucl. Phys. B 289 (1987) 847.

[3] L. McLerran, R. Venugopalan, Phys. Rev. D 49 (1994) 2233; D 49 (1994) 3352; D 50 (1994) 2225.

[4] J. Jalilian-Marian, A. Kovner, L. McLerran, H. Weigert, Phys. Rev. D 55 (1997) 5414.

[5] K.J. Eskola, K. Kajantie, P.V. Ruuskanen, K. Tuominen, Nucl. Phys. B 570 (2000) 379.

[6] A.H. Mueller, Nucl. Phys. B 572 (2000) 227.

[7] A.H. Mueller, Phys. Lett. B 475 (2000) 220.

[8] J. Bjoraker, R. Venugopalan, hep-ph/0008294.

[9] T.S. Biró, E. van Doorn, B. Müller, M.H. Thoma, X.-N. Wang, Phys. Rev. C 48, 1275 (1993).

[10] L. Xiong, E. Shuryak, Phys. Rev. C 49 (1994) 2203.

[11] S.M. Wong, Phys. Rev. C 54 (1996) 2588.

[12] D.M. Elliott, D.H. Rischke, Nucl. Phys. A 671 (2000) 583.

[13] K. Geiger, B. Müller, Nucl. Phys. B 369 (1992) 600.

[14] K. Geiger, Phys. Rep. 258 (1995) 237.

[15] A. Krasnitz, R. Venugopalan, Phys. Rev. Lett. 84 (2000) 4309; hep-ph/0007108.

[16] T.S. Biró, B. Müller, X.-N. Wang, Phys. Lett. B 283 (1992) 171.

[17] G.C. Nayak, A. Dumitru, L. McLerran, W. Greiner, hep-ph/0001202.

[18] J.F. Gunion, G. Bertsch, Phys. Rev. D 25 (1982) 746.

[19] R. Baier, Yu.L. Dokshitzer, A.H. Mueller, D. Schiff, Nucl. Phys. B 531 (1998) 403.

[20] R. Baier, D. Schiff, B.G. Zakharov, hep-ph/0002198.

[21] B.G. Zakharov, JETP Lett. 63 (1996) 952; 65 (1997) 615.

[22] J.D. Bjorken, Phys. Rev. D 27 (1983) 140.

[23] G. Baym, Phys. Lett. B 138 (1984) 18. 